\title{
Photoinduced Orientation of Photo-Cross-Linkable Liquid Crystalline Copolymer Films Comprised of H-Bonded and Non-H-Bonded Mesogenic Side Groups
}

\author{
By Nobuhiro KAWATSUKI, ${ }^{*}$ Mami NOBUHITANI, and Mizuho Kondo
}

The cooperative molecular reorientation in methacrylate copolymer films with hexamethylene spacer groups terminated with photoreactive hydrogen $(\mathrm{H})$ bonded 4-oxycinnamic acid (CA) and photoinactive 4-oxy-4'-methoxybiphenyl (MB) in their side chains was investigated by irradiating with linearly polarized ultraviolet light (LPUV) and subsequent annealing. A thermally enhanced cooperative in-plane orientation of both CA and MB side groups perpendicular to the polarization $(\boldsymbol{E})$ of LPUV light was obtained when the annealing temperature was in the liquid crystalline temperature range of both mesogenic side group. On the other hand, thermal enhancement of the in-plane molecular reorientation did not occur when the annealing temperature was out of the LC temperature range of the MB. It was clarified that the amount of axis-selectively photoreacted H-bonded CA groups and the LC mesomorphism of the copolymer film play an important role in the thermally enhanced cooperative molecular reorientation.

KEY WORDS: Photo-Cross-Linkable Polymer / Photoinduced Orientation / Hydrogen Bond / Polymer Liquid Crystal / Birefringent Film /

Axis-Selective Photoreaction /

The photo-control of molecular orientation in polymeric films that causes a large optical anisotropy of the film can be applicable to birefringent optical devices, the photoalignment layer of liquid crystal displays, optical memories, and holographic data storage devices. ${ }^{1-3}$ It is well known that the irradiating with linearly polarized (LP) light easily creates a small optical anisotropy in a photoreactive film based on an axis-selective photoreaction, and a large optical anisotropy is obtained when the axis-selective photoreaction is accompanied by a molecular reorientation. ${ }^{3-5}$ Various types of azobenzenecontaining polymeric materials and photo-cross-linkable polymer liquid crystalline (PPLC) materials containing cinnamate groups have been reported to achieve highly molecular reoriented films. ${ }^{3-7}$ Cooperative photoinduced molecular reorientation is also investigated by the copolymerization with comonomers comprised of non-photoreactive mesogenic side groups. ${ }^{8-11}$ The functionality of the reoriented films is derived from the cooperative reorientation, while the controllability of the molecular reorientation behavior is due to tuning of the comonomer.

During the course of our systematic study on PPLCs that show a photoinduced molecular orientation, we have found that a polymethacrylate comprised of a hexamethylene spacer group terminated with a 4-oxycinnamic acid (CA) in its side chain (P6CA) reveals a large photoinduced molecular reorientation with a high photoreactivity towards LPUV light. ${ }^{12} \mathrm{~A}$ LC mesomorphism P6CA is caused by the hydrogen $(\mathrm{H})$ bonded dimers of CA side groups. ${ }^{12-15}$ Furthermore, methacrylate copolymers having $\mathrm{CA}$ and 4-oxybenzoic acid (BA) side groups show the similar LC mesomorphism to P6CA with wide range of the copolymerization ratio due to the randomly- formed CA-CA, BA-BA, and CA-BA H-bonded mesogenic structures, and the cooperative photoinduced molecular reorientation is achieved to demonstrate a controllability of the photoinduced birefringence of the film by tuning the copolymer composition. ${ }^{16}$ However, a methacrylate monomer with CA side groups has not be copolymerized with comonomers comprised of non-H-bonded mesogenic side groups so far, which can expand the functionality of the P6CA-based LC polymers when the cooperative molecular reorientation is generated. In this paper, we synthesized methacrylate copolymers that contain CA and 4-oxy-4'-methoxybiphenyl (MB) side groups, which reveal LC mesomorphism without H-bonds. The influence of the copolymerization ratio on the thermal properties and the photoinduced molecular reorientation behavior of the copolymer films were investigated by polarization UV spectroscopy.

\section{EXPERIMENTAL}

\section{Materials}

Figure 1 shows chemical structure of (co)polymers P1aP1e used in this study. Methacrylate monomers with CA and MB side groups were synthesized according to the literatures. ${ }^{12,17}$ Copolymers were synthesized by a radical polymerization in THF solution using AIBN as an initiator. Adjusting the monomer feed ratio controlled copolymer composition. Table I summarizes composition, molecular weight, and thermal property of copolymers.

\section{Characterization}

Thermal properties were examined using a polarization 


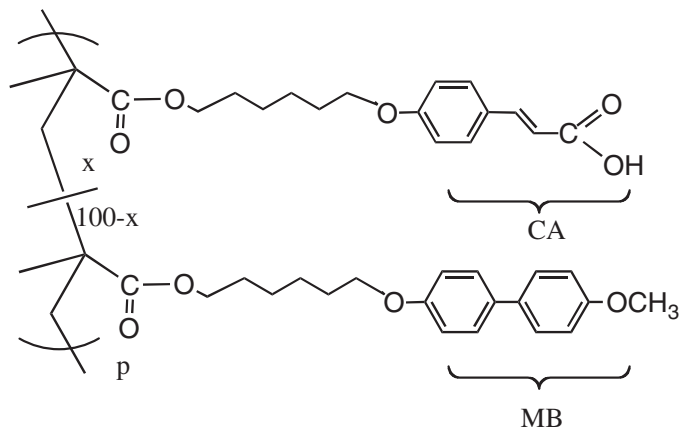

Figure 1. Chemical structure of copolymer used in this study

Table I. Composition, molecular weight, and thermal properties of (co)polymers

\begin{tabular}{crccl}
\hline Polymer & $\mathrm{x}^{\mathrm{a})}$ & $\left.M_{\mathrm{w}} \times 10^{-4} \mathrm{~b}\right)$ & $M_{\mathrm{w}} / M_{\mathrm{n}}$ & Thermal property ${ }^{\mathrm{c})}\left({ }^{\circ} \mathrm{C}\right)$ \\
\hline P1a & 100 & 3.9 & 1.5 & $\mathrm{G} 135 \mathrm{~N} \mathrm{187 \textrm {I }}$ \\
P1b & 75 & 4.6 & 1.6 & $\mathrm{G} 124 \mathrm{LC}_{1} 155 \mathrm{LC}_{2} 165 \mathrm{I}$ \\
P1c & 50 & 6.0 & 2.0 & $\mathrm{G} 102 \mathrm{LC}_{1} 140 \mathrm{LC}_{2} 147 \mathrm{I}$ \\
P1d & 25 & 10.5 & 2.3 & $\mathrm{G} 81 \mathrm{LC}_{1} 118 \mathrm{LC}_{2} 135 \mathrm{I}$ \\
P1e & 0 & 5.5 & 2.5 & $\mathrm{~K} 117 \mathrm{~S} 138 \mathrm{I}$
\end{tabular}

a) Determined by ${ }^{1} \mathrm{H}$ NMR . b) Measured by GPC with polystyrene standards. c) Determined by DSC and POM. K: crystalline, G: glassy, N: nematic, S: smectic, $\mathrm{LC}_{1}$ : LC phase with $\mathrm{MB}$ and $\mathrm{H}$-bonded $\mathrm{CA}$ groups, $\mathrm{LC}_{2}$ : LC phase with $\mathrm{H}$-bonded CA groups, I: isotropic.

optical microscope (POM) and a differential scanning calorimetry (DSC) analyzer. Polarization UV-vis spectra were measured using a spectrometer equipped with Glan-Taylor polarizing prisms. The in-plane order parameter, $S$, is expressed in the form of eq 1 ;

$$
S=\frac{A_{\|}-A_{\perp}}{A_{\text {(large })}+2 A_{(\text {small })}},
$$

where $A_{\|}$and $A_{\perp}$ are the absorbances parallel and perpendicular to $\boldsymbol{E}$, respectively, and $A_{\text {(large) }}$ is the larger value of $A_{\|}$and $A_{\perp}$, and $A_{\text {(small) }}$ is the smaller one. ${ }^{10} S$ was calculated by polarized UV-vis spectroscopy using wavelengths of $270 \mathrm{~nm}$ (MB groups) and $314 \mathrm{~nm}$ (CA groups). Birefringence of the reoriented films was measured by the Senarmont method at $633 \mathrm{~nm}$

\section{Photoreaction}

Thin films were prepared by spin-coating a THF solution of copolymers $(1.4 \mathrm{wt} / \mathrm{wt}-\%)$ onto a quartz substrate. The film thickness was approximately $0.2 \mu \mathrm{m}$. The film was irradiated by light from a $250 \mathrm{~W}$ high-pressure $\mathrm{Hg}-\mathrm{UV}$ lamp that was passed through Glan-Taylor polarizing prisms with a cut-off filter below $290 \mathrm{~nm}$. The light intensity was $10 \mathrm{~mW} / \mathrm{cm}^{2}$ at $365 \mathrm{~nm}$. Degree of the photoreaction (DP) was estimated by monitoring an absorption band at $314 \mathrm{~nm}$ (CA groups) of the UV spectra. For the thermally enhanced molecular reorientation, the irradiated film was annealed at elevated temperatures for $10 \mathrm{~min}$. The generated optical anisotropy of the film was measured by polarizing microscopy and polarization UV-vis spectroscopy.

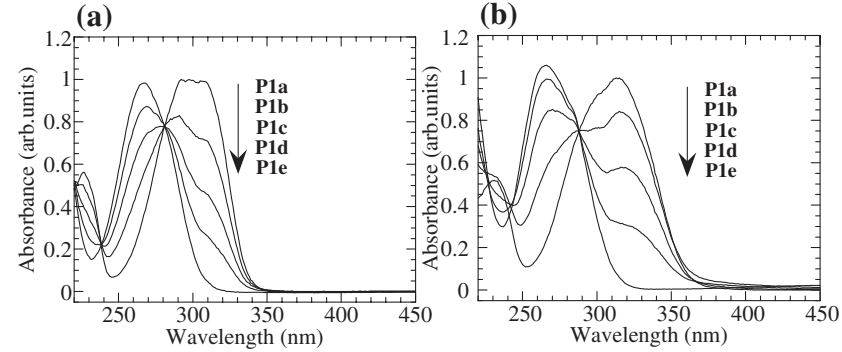

Figure 2. UV-vis absorption spectra of copolymer (a) in THF solution, and (b) films on quartz substrates.

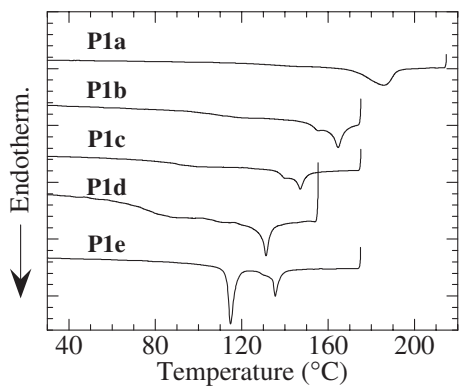

Figure 3. DSC second heating curves of copolymers.

\section{RESULTS AND DISCUSSION}

\section{Thermal and Spectroscopic Properties of Copolymers}

All copolymers shown in Figure 1 were synthesized by free radical copolymerization. Adjusting the feed ratio of the two methacrylate monomers controlled the copolymerization ratio. Table I summarizes the molecular weights of synthesized (co)polymers. Figure $2 \mathrm{a}$ and $2 \mathrm{~b}$ show the UV-vis absorption spectra of the (co)polymer in THF solution and thin films on quartz substrates. They reveal two absorption maxima, $270 \mathrm{~nm}$ and around $314 \mathrm{~nm}$, which correspond to the absorption bands of the $\mathrm{MB}$ and $\mathrm{CA}$ groups, respectively.

All synthesized (co)polymers show LC phase under the POM observation. Figure 3 shows the DSC scan of the (co)polymers. The copolymerization of the comonomer with MB side groups lowered the clearing point $\left(T_{\mathrm{i}}\right)$ due to the lower $T_{\mathrm{i}}$ of homopolymer P1e, indicating a random copolymerization of two monomers. Although the homopolymers (P1a and P1e) showed one nematic LC phase under POM observation and DSC scan, the DSC scan for copolymers (P1b-P1d) exhibited two endothermic peaks as shown in Figure 3. POM observation for these copolymers revealed that there were not specific differences between these temperature ranges. This means that the copolymers revealed two types of LC phases as summarized in Table I; the lower LC temperature range $\left(\mathrm{LC}_{1}\right)$ was due to both the $\mathrm{H}$-bonded $\mathrm{CA}$ groups and the MB mesogenic groups. The disassociation of the LC characteristics of the $\mathrm{MB}$ side groups occurred in the higher LC temperature range $\left(\mathrm{LC}_{2}\right)$, where a sufficient amount of the $\mathrm{H}$-bonded $\mathrm{CA}$ side groups was still available to exhibit the 

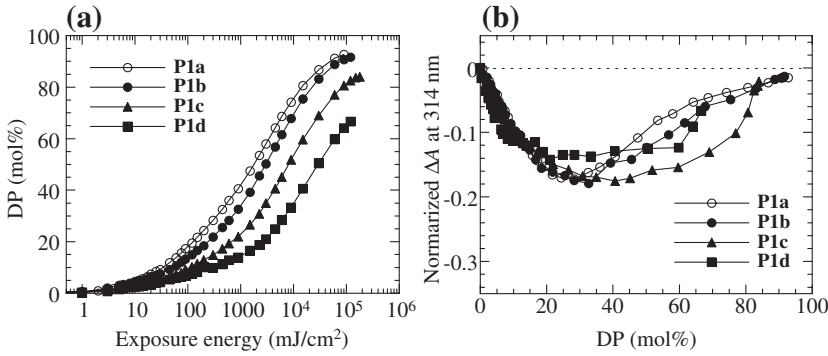

Figure 4. a) Degree of the photoreaction (DP) of copolymer films as a function of exposure energy. (b) Normalized photoinduced optical anisotropy $(\triangle A)$ of copolymer films at $314 \mathrm{~nm}$ as a function of DP. Initial absorbance at $314 \mathrm{~nm}$ is normalized to 1.0 .

nematic LC phase of the material. Namely, both mesogenic groups independently showed LC mesomorphism. Detailed LC phases should be determined by XRD analysis. Similar thermal properties were observed in LC copolymers comprised of $\mathrm{H}$ bonded benzoic acid side groups and non-H-bonded mesogenic side groups. ${ }^{18}$

\section{Axis-selective Photoreaction of Copolymer Films}

Irradiating a copolymer film with LPUV light induced an axis-selective $[2+2]$ photodimerization and a photoisomerization reaction of the CA groups. All copolymer films became insoluble after exposure, indicating the photo-cross-linking. Figure 4a plots degree of the photoreaction (DP) of the CA groups as a function of exposure energy. It reveals that the rates of the photoreaction decreased when the content of MB groups increased. This is because that the $[2+2]$ photodimerization reaction becomes difficult when the content of CA groups decreased due to the increased distance between two cinnamic acids. $^{16}$ Because the photoreaction of CA groups in the direction parallel to $\boldsymbol{E}$ of LPUV light is faster than that perpendicular to $\boldsymbol{E}$, a small negative optical anisotropy ( $\triangle A=A_{\|}-A_{\perp}<0$ ) of the film is generated after the photoreaction. ${ }^{12}$ Figure $4 \mathrm{~b}$ plots the photoinduced $\Delta A$ at $314 \mathrm{~nm}$, where the absorbance of the initial film was normalized one, as a function of DP. Because the molecular reorientation in the copolymer films rarely occurred during the exposure, $\Delta A$ values were similar to each other, and maximum $\Delta A$ s were obtained when the DP was approximately $30-50 \mathrm{~mol} \%$ for all the films.

Thermal Amplification of Photoinduced Optical Anisotropy

We have previously reported that a small photoinduced $\Delta A$ of a P1a film was thermally amplified perpendicular to $\boldsymbol{E}$ of LPUV light by annealing the exposed film in the LC temperature range when the DP was around 2-4 mol \%, and the efficient molecular reorientation was obtained when the annealing temperature was close to the clearing point of the material. ${ }^{12}$ For the copolymers P1b-P1d, thermal amplification of the negative photoinduced optical anisotropy was observed, but its behavior differed from that of P1a.

Figure 5a-5c show the UV-vis polarization spectra of copolymer films before photoirradiating, after irradiating, and
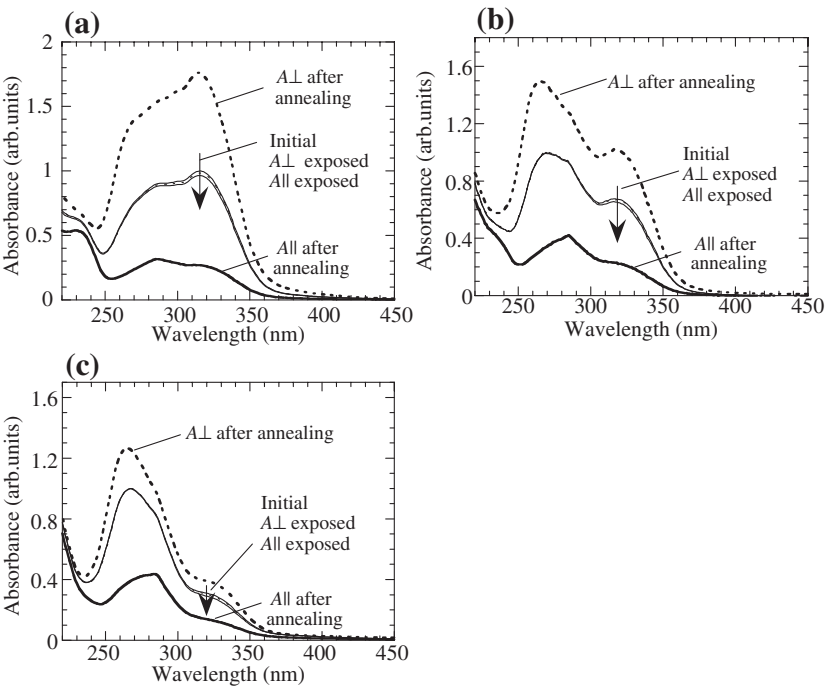

Figure 5. UV-vis polarization spectra of copolymer films before photoirradiating, after irradiating (thin lines), and after subsequent annealing (thick lines) for $10 \mathrm{~min}$. Solid line represent $A_{\|}$, while dotted lines show $A_{\perp}$. (a) $\mathbf{P} 1 \mathbf{b}$, irradiating with $5 \mathrm{~mJ} \mathrm{~cm}^{-2}$ doses and annealed at $140^{\circ} \mathrm{C}$; (b) P1c, irradiating with $7 \mathrm{~mJ} \mathrm{~cm}^{-2}$ doses and annealed at $130^{\circ} \mathrm{C}$; (c) $\mathbf{P 1 d}$, irradiating with $10 \mathrm{~mJ} \mathrm{~cm}^{-2}$ doses and annealed at $115^{\circ} \mathrm{C}$.

after subsequent annealing at the $\mathrm{N}_{1}$ temperature range of the copolymers. The annealing temperatures were $140^{\circ} \mathrm{C}$ for $\mathbf{P 1 b}$, $130{ }^{\circ} \mathrm{C}$ for P1c, and $115^{\circ} \mathrm{C}$ for P1d. The DP of the films was approximately $2-5 \mathrm{~mol} \%$. After the LPUV photoreaction, a small negative $\Delta A$ was observed around $314 \mathrm{~nm}$ due to the axis-selective photoreaction of the H-bonded CA groups. For all the copolymer films, the annealing process enhanced the negative $\triangle A$ and generated the cooperative molecular reorientation for both the $\mathrm{H}$-bonded $\mathrm{CA}$ and $\mathrm{MB}$ side groups perpendicular to $\boldsymbol{E}$ of LPUV light. The enhanced $S$ values at $270 \mathrm{~nm}$ and at $314 \mathrm{~nm}$ were -0.62 and -0.65 for P1b, -0.52 and -0.53 for P1c, and -0.48 and -0.43 for P1d, respectively. This means that a small amount of the photoreacted CA groups initiated the thermally amplified cooperative molecular reorientation for both $\mathrm{CA}$ and $\mathrm{BA}$ side groups. The annealing procedure generates the self-organization of all the LC mesogenic groups in a perpendicular direction due to its higher LC characteristics where a small amount of photoreacted CA groups parallel to $\boldsymbol{E}$ acted as impurities, which reduce the LC mesophorism in the parallel direction. ${ }^{12,16}$ The generated birefringence values $(\Delta n)$ of the copolymer films were 0.16 for P1b, 0.14 for P1c and 0.12 for P1d. The birefringence of P1b film is higher than that of homopolymer P1a $(\Delta n=0.15)$ because of higher inherent birefringence of $\mathrm{MB}$ groups than that of CA. However, the lower birefringence values for the P1c and P1d films were due to the lower in-plane order of the reoriented films. Small $S$ values for P1c and P1d are because that the large amount of MB groups inhibited the effective inplane molecular reorientation, where the thermal process generated the out-of-plane orientation of the MB side groups. ${ }^{17}$

On the other hand, when the exposed films (DP $=2-3$ mol \%) were annealed in the $\mathrm{N}_{2}$ temperature range of the 

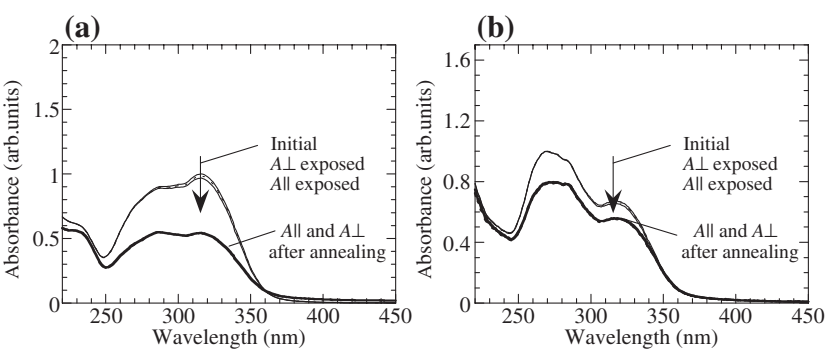

(c)

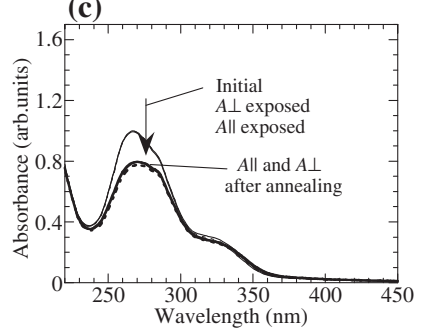

Figure 6. UV-vis polarization spectra of copolymer films before photoirradiating, after irradiating (thin lines), and after subsequent annealing (thick lines) for $10 \mathrm{~min}$. Solid line represent $A_{\|}$, while dotted lines show $A_{\perp}$. (a) $\mathbf{P 1 b}$, irradiating with $5 \mathrm{~mJ} \mathrm{~cm}^{-2}$ doses and annealed at $160^{\circ} \mathrm{C}$; (b) P1c, irradiating with $7 \mathrm{~mJ} \mathrm{~cm}^{-2}$ doses and annealed at $150^{\circ} \mathrm{C}$; (c) P1d, irradiating with $10 \mathrm{~mJ} \mathrm{~cm}^{-2}$ doses and annealed at $130^{\circ} \mathrm{C}$.

copolymers, molecular reorientation did not occur as shown in Figure 6 , where the annealing temperatures were $160^{\circ} \mathrm{C}$ for $\mathbf{P 1 b}, 150^{\circ} \mathrm{C}$ for $\mathbf{P 1 c}$, and $130^{\circ} \mathrm{C}$ for $\mathbf{P 1 d}$, respectively. At these temperatures, the $\mathrm{MB}$ groups in the copolymers did not reveal the LC characteristics although the materials still showed the nematic character due to the $\mathrm{H}$-bonded $\mathrm{CA}$ groups as described in above section. The MB groups, which were in the isotropic state, inhibited the thermally amplified in-plane molecular reorientation of the mesogenic $\mathrm{H}$-bonded CA groups. Additionally, the decrease in the absorbances in both parallel and perpendicular direction after the annealing indicates the thermally enhanced out-of-plane reorientation of the mesogenic side groups. ${ }^{17}$

The influence of the annealing temperature on the thermal amplification of the photoinduced negative $\Delta A$ of the film was investigated in detail. Figure 7 plot the $S$ values of the copolymer films annealed at various temperatures when the DP was approximately $2 \mathrm{~mol} \%$. The maximum $S$ values were attained when the annealing temperature was $10-15^{\circ} \mathrm{C}$ lower than the $\mathrm{N}_{1}-\mathrm{N}_{2}$ transition temperature, and the annealing in the $\mathrm{N}_{2}$ temperature range did not generate the in-plane orientation at all. Figure 8 plot the thermally enhanced $S$ values as a function of the DP. It shows that the thermal amplification of the photoinduced negative $\Delta A$ was generated for all the copolymers at the early stage of the photoreaction (DP $=1-5 \mathrm{~mol} \%$ ). When the exposed film with the DP around 10-20 mol \% was annealed at the LC temperature range of the film, the photoinduced $\Delta A$ was reversely enhanced parallel to the polarization of LPUV light although the generated $S$ values were less than +0.15 . This is because that the axis-selectively photoreacted H-bonded CA-CA side groups act as photo-cross-

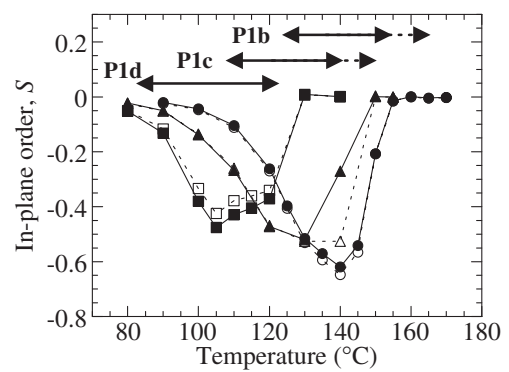

Figure 7. Thermally enhanced $S$ values of the photoreacted copolymer films when the annealing temperature is varied. Circles; $\mathbf{P} 1 \mathbf{b}$, triangles; P1c, squares; P1d. Closed and open points represent $S$ at $270 \mathrm{~nm}$ and $S$ at $314 \mathrm{~nm}$, respectively. Arrows exhibit the LC temperature range of materials. Degrees of the photoreaction are $2 \mathrm{~mol} \%$.

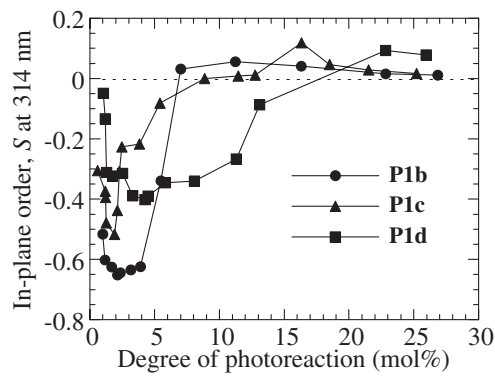

Figure 8. Thermally enhanced $S$ values at $314 \mathrm{~nm}$ of the photoreacted copolymer films as a function of the degree of the photoreaction. Circles; P1b, triangles; P1c, squares; P1d. Annealing temperatures are $140^{\circ} \mathrm{C}$ for $\mathbf{P} 1 \mathbf{b}, 130^{\circ} \mathrm{C}$ for $\mathbf{P} 1 \mathbf{c}, 115^{\circ} \mathrm{C}$ for $\mathbf{P} 1 \mathbf{d}$.

linked anchors to induce the in-plane molecular reorientation parallel to $\boldsymbol{E} .^{12}$ These behaviors are similar to that of the P1a film when the DP is around $15 \mathrm{~mol} \%{ }^{12}$

\section{CONCLUSIONS}

Methacrylate copolymers comprised of photo-cross-linkable $\mathrm{H}$-bonded $\mathrm{CA}$ side groups and non-H-bonded MB side groups were synthesized and characterized. All copolymers revealed two types of nematic LC phases; one is a nematic LC phase due to the $\mathrm{H}$-bonded $\mathrm{CA}$ and $\mathrm{MB}$ side groups $\left(\mathrm{N}_{1}\right.$ phase) and another is due to the $\mathrm{H}$-bonded CA groups $\left(\mathrm{N}_{2}\right.$ phase). Irradiating with the LPUV light resulted in small photoinduced optical anisotropy of the film, and the thermally amplified cooperative molecular reorientation of both mesogenic side groups was generated when the exposed film was annealed in the $\mathrm{N}_{1} \mathrm{LC}$ temperature range. However, the amplification did not occur when the exposed film was annealed in the $\mathrm{N}_{2} \mathrm{LC}$ temperature range. The selection of the annealing temperature to exhibit the LC characteristics for both mesogenic side groups plays an important role in the cooperative molecular reorientation. The reoriented films can be applicable to the birefringent optical films, in which adjusting the copolymerization ratios controls the birefringence and the annealing temperature for the effective molecular reorientation. 
Acknowledgment. This work was partly supported by Grantin-Aid for Scientific Research in Priority Areas "New Frontiers in Photochromism (No. 471)" from the Ministry of Education, Culture, Sports, Science and Technology (MEXT), and Grantin-Aid for Scientific Research (B, No. 21350129 and S, No. 21225006) from Japan Society for the Promotion of Science.

Received: June 17, 2009

Accepted: July 15, 2009

Published: August 26, 2009

\section{REFERENCES}

1. V. P. Shibaev, S. G. Kostromin, and S. A. Ivanov, in "Polymers as Electroactive and Photooptical Media," V. P. Shibaev, Ed., Springer, Berlin, 1996, p 37.

2. P. O. Jackson, M. O'Neill, W. Duffy, W. L. Hindmarsh, S. M. Kelly, and G. J. Owen, Chem. Mater., 13, 694 (2001).

3. a) K. Anderle, R. Birenheide, M. Eich, and J. H. Wendrorff, Makromol. Chem. Rapid Commun., 10, 477 (1989). b) T. Ikeda, J. Mater. Chem., 13, 2037 (2003).

4. N. Kawatsuki, H. Takatsuka, T. Yamamoto, and O. Sangen, J. Polym. Sci., Part A: Polym. Chem., 36, 1521 (1998).
5. N. Kawatsuki, H. Ono, H. Takatsuka, T. Yamamoto, and O. Sangen, Macromolecules, 30, 6680 (1997).

6. a) Y. Wu, Y. Demachi, O. Tsutsumi, A. Kanazawa, T. Shiono, and T. Ikeda, Macromolecules, 31, 349 (1998).

b) Y. Wu, Y. Demachi, O. Tsutsumi, A. Kanazawa, T. Shiono, and T. Ikeda, Macromolecules, 31, 1104 (1998).

7. N. Kawatsuki, K. Goto, T. Kawakami, and T. Yamamoto, Macromolecules, 35, 706 (2002).

8. Y. Wu, Q. Zhang, A. Kanazawa, T. Shiono, T. Ikeda, and Y. Nagase, Macromolecules, 32, 3951 (1999).

9. L. Läsker, Th. Fischer, J. Stumpe, S. G. Kostromin, S. Ivanov, V. Shivaev, and R. Ruhmann, Mol. Cryst. Liq. Cryst., 246, 347 (1994).

10. N. Kawatsuki, C. Suehiro, and T. Yamamoto, Macromolecules, 31, 5984 (1998).

11. J. Lee and N. Kawatsuki, Mol. Cryst. Liq. Cryst., 498, 59 (2009).

12. E. Uchida and N. Kawatsuki, Macromolecules, 39, 9357 (2006).

13. T. Kato and J. M. Fréchet, J. Am. Chem. Soc., 111, 8533 (1989).

14. U. Kumar, T. Kato, and J. M. Fréchet, J. Am. Chem. Soc., 114, 6630 (1992).

15. T. Kato, N. Mizoshita, and K. Kanie, Macromol. Rapid Commun., 22, 797 (2001).

16. N. Kawatsuki, T. Kawanishi, and E. Uchida, Macromolecules, 41, 4642 (2008).

17. N. Kawatsuki, N. Furuso, K. Goto, and T. Yamamoto, Macromol. Chem. Phys., 203, 2438 (2002).

18. N. Kawatsuki and E. Uchida, Polymer, 48, 3066 (2007). 